\title{
Coronary CT angiography and myocardial perfusion imaging to detect flow-limiting stenoses: a potential gatekeeper for coronary revascularization?
}

\author{
Oliver Gaemperli', Lars Husmann ${ }^{1}$, Tiziano Schepis ${ }^{1}$, Pascal Koepfli ${ }^{1}$, Ines Valenta ${ }^{1}$, \\ Walter Jenni ${ }^{1}$, Hatem Alkadhi ${ }^{2}$, Thomas F. Lüscher ${ }^{1}$, and Philipp A. Kaufmannn ${ }^{1,3 *}$
}

${ }^{1}$ Cardiac Imaging, University Hospital Zurich, Ramistrasse 100, CH-8091 Zurich, Switzerland; ${ }^{2}$ Institute of Diagnostic Radiology, University Hospital Zurich, Zurich, Switzerland; and

${ }^{3}$ Zurich Center for Integrative Human Physiology (ZIHP), University of Zurich, Zurich, Switzerland

Received 17 November 2008; revised 5 May 2009; accepted 30 June 2009; online publish-ahead-of-print 14 August 2009

This paper was guest edited by Prof. Van de Werf, Department of Cardiology, University Hospitals Leuven, Belgium

\begin{abstract}
Aims
To evaluate the diagnostic accuracy of a combined non-invasive assessment of coronary artery disease with coronary CT angiography (CTA) and myocardial perfusion imaging (MPI) for the detection of flow-limiting coronary stenoses and its potential as a gatekeeper for invasive examination and treatment.

Methods In 78 patients (mean age $65 \pm 9$ years) referred for coronary angiography (CA), additional CTA and MPI (using singleand results photon emission-computed tomography) were performed and the findings not communicated. Detection of flowlimiting stenoses (justifying revascularization) by the combination of CTA and MPI (CTA/MPI) was compared with the combination of quantitative coronary angiography (QCA) plus MPI (QCA/MPI), which served as standard of reference. The findings of both combinations were related to the treatment strategy (revascularization vs. medical treatment) chosen in the catheterization laboratory based on the CA findings. Sensitivity, specificity, positive and negative predictive value, and accuracy of CTA/MPI for the detection of flow-limiting coronary stenoses were $100 \%$ each. More than half of revascularization procedures $(21 / 40,53 \%)$ was performed in patients without flow-limiting stenoses and $76 \%$ (47/62) of revascularized vessels were not associated with ischaemia on MPI.

Conclusion

The combined non-invasive approach CTA/MPI has an excellent accuracy to detect flow-limiting coronary stenoses compared with QCA/MPI and its use as a gatekeeper appears to make a substantial part of revascularization procedures redundant.

Keywords Coronary CT angiography • Myocardial perfusion imaging • Conventional coronary angiography • Coronary artery disease
\end{abstract}

\section{Introduction}

Over the past decades, many advances in imaging techniques have enhanced our pathophysiologic understanding of coronary artery disease (CAD). A comprehensive assessment of CAD should include both information on coronary artery anatomy and functional information about the haemodynamic relevance of coronary artery lesions in order to guide revascularization procedures. ${ }^{1-3}$
In stable CAD, the debate on the role of elective percutaneous coronary intervention $(\mathrm{PCl})$ is highly controversial. ${ }^{4,5}$ Guidelines recommend proof of ischaemia prior to elective revascularization of coronary stenoses, ${ }^{2,4,6}$ and several reports have demonstrated that $\mathrm{PCl}$ fails to improve prognosis in patients with stable CAD compared with conservative treatment. ${ }^{5,7}$ Nonetheless, in clinical practice, the decision to revascularize is often based solely upon visual angiographic criteria rather than objective proof of

* Corresponding author. Tel: +41 4425535 55, Fax: +41 4425544 14, Email: pak@usz.ch

Published on behalf of the European Society of Cardiology. All rights reserved. (C) The Author 2009. For permissions please email: journals.permissions@oxfordjournals.org. 
ischaemia. Similarly, while quantitative coronary angiography (QCA) is commonly used as gold standard in clinical trials, ${ }^{8}$ its clinical role is limited as accurate analysis is generally not readily available during the procedure.

Coronary multislice CT angiography (CTA) has evolved rapidly during the past decade allowing now visualization of coronary artery morphology and lesions with a temporal and spatial resolution that approaches conventional coronary angiography (CA). ${ }^{9,10}$ Combination of CTA and myocardial perfusion imaging (MPI) is non-invasive, and thus, allow non-invasive integrative assessment of CAD. ${ }^{11}$ Hence, it seems conceivable that an early non-invasive assessment of CAD with CTA and SPECT may act as a gatekeeper for conventional coronary angiography and thus avoid unnecessary invasive diagnostic and revascularization procedures.

Therefore, the aim of the present study was to evaluate the diagnostic accuracy of the combination CTA/MPI vs. QCA/MPI as a gatekeeper for invasive coronary examination and treatment.

\section{Methods}

\section{Study population and study design}

We prospectively enrolled consecutive patients with known or suspected CAD referred to our institution for elective CA. The clinical decision to perform CA was based on the history and/or symptoms of the patient and/or on the results from exercise stress testing. Patients were eligible if they were in a stable clinical condition i.e. if they were in Canadian Cardiac Society class I to III, and in New York Heart Association functional class I to III. Exclusion criteria were severe obstructive lung disease, high-grade atrioventricular conduction disturbances, atrial fibrillation, and known intolerance of iodinated contrast agents. Patients who agreed to participate underwent CTA and myocardial perfusion SPECT prior to the invasive procedure and the results from non-invasive testing were withheld from the interventional cardiologist. The study protocol was approved by the local institutional review board and all patients gave written informed consent before enrolment. All patients were made aware of the additional radiation dose from MPI and CTA prior to consent. The study population is shared with a prior publication by our group. ${ }^{12}$

\section{CT angiography image acquisition}

All scans were performed on a 64-slice CT scanner (Somatom Sensation 64; Siemens Medical Solutions, Forchheim, Germany). Patients with pre-scan heart rates above 70 b.p.m. received intravenous betablocker therapy (5-15 mg metoprolol) immediately prior to the CT scan. Low-dose calcium score, helical CTA scanning, and image reconstruction parameters were used as previously reported. ${ }^{12,13}$

\section{CT angiography image interpretation}

CT angiography image interpretation was performed on axial source images, multiplanar and curved reformations, and thin-slab maximum intensity projections. First, image quality for each data set was rated by one reader on a scale ranging from score 1 (excellent image quality), 2 (good image quality), 3 (moderate image quality), 4 (poor, but still diagnostic image quality), to score 5 (very poor image quality, non-evaluable data set defined as having at least one non-evaluable segment). Coronary arteries were subdivided according to a 15-segment model proposed by the American Heart Association. ${ }^{14}$
Then, each segment was visually evaluated on at least two planes, one parallel and one perpendicular to the course of the vessel with regard to coronary artery delineation. On these images, the degree of diameter stenosis was qualitatively graded by two independent readers (who were both blinded to the clinical history and to the findings from MPI and CA) on a decimal scale in $10 \%$ steps from 0 to $100 \%$. The ultimate diameter stenosis was calculated as the mean of both measurements. A significant stenosis was defined as narrowing of the coronary lumen $\geq 50 \%$, and all vessels with a diameter down to $1.5 \mathrm{~mm}$ were included in the analysis.

\section{Myocardial perfusion imaging image acquisition}

SPECT image acquisition was performed using a 1 day electrocardiographically (ECG) gated stress/rest protocol with adenosine stress $(140 \mu \mathrm{g} / \mathrm{kg} / \mathrm{min})$ and ${ }^{99 \mathrm{~m}} \mathrm{Tc}$-tetrofosmin $(250-350 \mathrm{MBq}$ at peak stress and three times the stress dose at rest according to standard protocol). ${ }^{15}$ Scanning parameters and image reconstruction algorithms were applied as previously reported. ${ }^{12}$ Patients were told to refrain from caffeine-containing beverages for at least $12 \mathrm{~h}$, nitrates and calcium channel blockers $24 \mathrm{~h}$, and beta-blockers $48 \mathrm{~h}$ before the MPI study.

\section{Myocardial perfusion imaging image interpretation}

SPECT image interpretation was visually performed by consensus of two experienced nuclear cardiologists on short axis, horizontal long axis, and vertical long-axis slices, and semiquantitative polar maps of perfusion using previously validated automated software. ${ }^{16}$ Anterior and septal wall perfusion defects were allocated to the left anterior descending (LAD) coronary artery, lateral defects to the left circumflex (LCX) coronary artery, and inferior defects to the right coronary artery (RCA). Reversible perfusion defects were considered to represent myocardial ischaemia. Fixed perfusion defects with concomitant regional wall motion abnormalities were considered to be myocardial scars. $^{17}$

\section{Quantitative coronary angiography}

Biplane conventional CA was performed according to standard techniques and evaluated by an experienced observer who was blinded to the results from CTA and MPI. Quantitative coronary angiography measurements were performed on two different image planes using an automated edge-detection system (Xcelera 1.2, Philips Medical Systems, Best, The Netherlands) as previously described. ${ }^{12} \mathrm{~A}$ significant stenosis was defined as a diameter reduction of $\geq 50 \%$.

\section{Comparison of CT angiography and quantitative coronary angiography}

The diagnostic accuracy of CTA was assessed by comparison with the results from QCA, which was considered to be the gold standard for coronary stenosis evaluation. Comparison was performed on an intention-to-diagnose basis and therefore, non-evaluable segments on CTA were considered as positives.

\section{Assessment of flow-limiting coronary stenoses}

A flow-limiting coronary stenosis was defined as a lesion with a diameter narrowing exceeding $50 \%$ (on CTA or on QCA) inducing a reversible perfusion defect in its subtending myocardial territory on MPI (myocardial ischaemia) (Figure 1). A coronary stenosis of $\geq 50 \%$ 


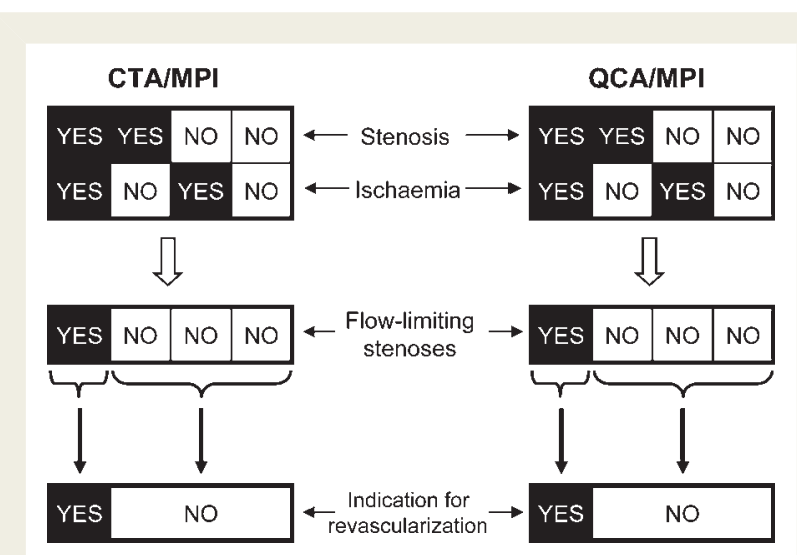

Figure I Diagnostic and treatment algorithm. A flow-limiting coronary stenosis (third row) was defined in the presence of angiographically significant stenoses (first row) and evidence of ischaemia on myocardial perfusion imaging (second row). Only flow-limiting coronary stenoses were considered as an indication for revascularization (fourth row).

without any associated myocardial ischaemia was considered to be non-flow-limiting. Conversely, a reversible perfusion defect in a territory subtended by a non-stenotic coronary artery was considered to represent a false-positive MPI result. As shown in Figure 1, the presence or absence of flow-limiting coronary stenosis was assessed independently for the combination of CTA plus MPI (CTA/MPI) and for the combination of QCA plus MPI (QCA/MPI), the latter being considered the gold standard for a combined assessment of coronary morphology and haemodynamic lesion severity.

\section{Coronary revascularizations}

Coronary revascularization procedures included $\mathrm{PCl}$ with or without stent implantation and coronary artery bypass grafting (CABG). According to guidelines, a stenosis was considered as an indication for revascularization only if it was associated with a reversible perfusion defect on MPI (flow-limiting stenosis). Finally, the patients' actual treatment strategy (revascularization vs. medical treatment) was compared with the imaging-derived treatment recommendations, on patient- and vessel-based analysis.

\section{Statistical analysis}

Statistical analysis was performed using the SPSS software package (SPSS 12.0.1 for Windows, SPSS Corp.). Quantitative data are expressed as mean \pm SD (unless otherwise stated) and categorical data given in proportions and percentages. Statistical comparison of quantitative data was performed using an unpaired two-tailed Student's $t$-test or Mann-Whitey $U$ test where appropriate and comparison of categorical data using a chi-squared test with Yates' correction or McNemar's test for comparison of paired proportions. A P-value $<0.05$ was considered statistically significant for all tests. Pre-test CAD likelihood was calculated according to Diamond and Forrester. ${ }^{18}$ Sensitivity, specificity, positive (PPV), and negative predictive value (NPV), and accuracy were obtained from $2 \times 2$ contingency tables and their respective $95 \%$ confidence intervals $(\mathrm{Cls})$ calculated from binomial expression. Accuracy was determined as the percentage of correct diagnoses in the entire sample. Univariate logistic regression was used to identify predictors for revascularization and the regression results are presented as odds ratios and their respective 95\% Cls. Additionally, multivariate logistic regression was applied to identify independent predictors by including all factors with $P<0.05$ and correction for the baseline characteristics with $P<0.1$ on univariate analysis.

\section{Results}

Ninety-six patients were enrolled in the study, of which 18 (19\%) had to be excluded: no CTA $(n=7)$ due to atrial fibrillation or technical reasons, no CA $(n=11)$ due to rescheduling, consent withdrawal, clinical deterioration, and logistic reasons. The final analysis included 78 patients with a mean age of $65 \pm 9$ years (range, 40-87 years) [35 (45\%) female] (Table 1). The median time interval between CTA and MPI was 0 days (range, 0 to 26 days), between CTA and CA 1 day (range, 0 to 22 days), and between MPI and CA 1 day (range, 0 to 26 days). A delay of more than 2 weeks between CTA and MPI was found in only one patient, and between CTA/MPI and CA in five patients.

\section{CT angiography results}

All patients were in stable sinus rhythm and the mean heart rate during the CT scan was $62 \pm 9$ b.p.m. Ten patients (13\%) were pre-treated with intravenous metoprolol.

A total number of 1093 coronary segments in 310 main coronary arteries were analysed. In two patients, the left main coronary artery (LMA) was missing as LAD and LCX had separate origins from the left coronary sinus. The mean image quality score was $2.9 \pm 0.9$. Image quality scores were 1 in 1 (1\%) patient, 2 in 31 (40\%) patients, 3 in 26 (33\%) patients, 4 in 15 (19\%) patients, and 5 in $5(6 \%)$ patients. Eight (1\%) coronary segments were not evaluable because of motion artefacts $(n=2)$, heavy calcifications $(n=1)$, or both $(n=5)$. On intention-to-diagnose basis, visual CTA image analysis revealed a stenosis in 137/1093 (13\%) segments corresponding to $91 / 310$ (29\%) coronary arteries in $46 / 78$ (59\%) patients (Figure 2). The details of the CTA results are given in Table 2. Interreader agreement for stenosis detection on CTA was $92 \%$ (95\% Cl, 90-93\%) on segment-based analysis.

\section{Myocardial perfusion imaging results}

Image quality of MPI was amenable to visual interpretation in all 78 patients. Visual image analysis revealed 14 reversible, 13 fixed, and 6 partially reversible perfusion defects in 31/78 (40\%) patients (Table 2). The distribution of the perfusion defects among the different coronary artery territories was as follows: reversible perfusion defects: $\operatorname{LAD}(n=8), \operatorname{LCX}(n=2), \operatorname{RCA}(n=4)$; fixed perfusion defects: LAD $(n=4), \operatorname{LCX}(n=3), \operatorname{RCA}(n=6)$; partially reversible perfusion defects: $\operatorname{LAD}(n=1), \operatorname{LCX}(n=1)$, $\operatorname{RCA}(n=4)$.

\section{Coronary angiography results}

Quantitative coronary angiography analysis of biplane CA revealed stenoses (of $\geq 50 \%$ diameter stenosis) in $92 / 310$ (30\%) coronary arteries corresponding to $49 / 78$ (63\%) patients. As with CTA, the LMA was missing in two patients as LAD and LCX had separate 
Table I Patient characteristics

\begin{tabular}{|c|c|c|c|c|}
\hline & All patients $(n=78)$ & Revasc group $(n=40)$ & Medical group $(n=38)$ & $P$-value* \\
\hline Age (years) & $65 \pm 9$ & $66 \pm 8$ & $63 \pm 10$ & 0.18 \\
\hline Female gender, $n(\%)$ & $35(45)$ & $13(33)$ & $22(58)$ & 0.02 \\
\hline Body mass index $\left(\mathrm{kg} / \mathrm{m}^{2}\right)$ & $26 \pm 4$ & $27 \pm 4$ & $26 \pm 4$ & 0.37 \\
\hline Systolic blood pressure (mmHg) & $134 \pm 19$ & $135 \pm 19$ & $132 \pm 19$ & 0.47 \\
\hline Diastolic blood pressure (mmHg) & $78 \pm 12$ & $77 \pm 11$ & $80 \pm 12$ & 0.35 \\
\hline Total serum cholesterol (mmol/L) & $4.8 \pm 1.0$ & $4.7 \pm 0.9$ & $4.9 \pm 1.1$ & 0.32 \\
\hline \multicolumn{5}{|l|}{ Cardiovascular history, n (\%) } \\
\hline Known CAD & $19(24)$ & $14(35)$ & $5(13)$ & 0.02 \\
\hline Single-vessel CAD & $5(6)$ & $3(8)$ & $2(5)$ & 0.69 \\
\hline Two-vessel CAD & $5(6)$ & $4(10)$ & $1(3)$ & 0.39 \\
\hline Three-vessel CAD & $9(12)$ & $7(18)$ & $2(5)$ & 0.18 \\
\hline Previous MI/ACS & $16(21)$ & $11(28)$ & $5(13)$ & 0.20 \\
\hline Previous $\mathrm{PCl}$ & $19(24)$ & $14(35)$ & $5(13)$ & 0.05 \\
\hline Previous CABG & $0(0)$ & $0(0)$ & $0(0)$ & - \\
\hline Missing exercise test & $34(44)$ & $19(48)$ & $15(39)$ & 0.63 \\
\hline Pathological exercise test & $35(45)$ & $16(40)$ & $19(50)$ & 0.51 \\
\hline $\operatorname{LVEF}(\%)$ & $59 \pm 15$ & $60 \pm 11$ & $59 \pm 18$ & 0.94 \\
\hline \multicolumn{5}{|l|}{ Symptoms, n (\%) } \\
\hline Angina pectoris CCS I-III & $30(38)$ & $21(53)$ & $9(24)$ & 0.02 \\
\hline Atypical chest pain & $16(20)$ & $5(13)$ & $11(29)$ & 0.13 \\
\hline Dyspnoea NYHA I-III & $19(24)$ & $7(18)$ & $12(32)$ & 0.24 \\
\hline None & $13(17)$ & $7(18)$ & $6(16)$ & $>0.99$ \\
\hline \multicolumn{5}{|l|}{ Cardiovascular risk factors, $n$ (\%) } \\
\hline Diabetes mellitus & $13(17)$ & $11(28)$ & $2(5)$ & 0.02 \\
\hline Hypertension & $61(78)$ & $34(85)$ & $27(71)$ & 0.22 \\
\hline Dyslipidaemia & $40(51)$ & $24(60)$ & $16(42)$ & 0.18 \\
\hline Current or former smokers & $40(51)$ & $25(63)$ & $15(39)$ & 0.07 \\
\hline Unknown CAD & $59(76)$ & $26(65)$ & $33(87)$ & 0.03 \\
\hline CAD pre-test likelihood (\%) & $75 \pm 26$ & $86 \pm 16$ & $66 \pm 30$ & $<0.01$ \\
\hline Framingham risk score & $12 \pm 9$ & $15 \pm 9$ & $10 \pm 7$ & 0.01 \\
\hline
\end{tabular}

Data not given in $n(\%)$ is shown as mean \pm SD.

Revasc group, group of patients undergoing coronary revascularization; Medical group, group of patients treated conservatively; CAD, coronary artery disease; MI, myocardial infarction; ACS, acute coronary syndrome; PCl, percutaneous coronary intervention; CABG, coronary artery bypass grafting; LVEF, left ventricular ejection fraction as assessed with gated SPECT; CCS, Canadian cardiac society; NYHA, New York Heart Association.

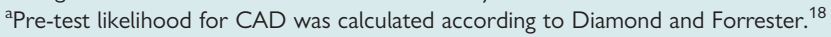

*P-value for comparison of Revasc vs. Medical group.

origins from the left coronary sinus. The details of the QCA results are given in Table 2.

\section{Comparison of CT angiography vs. quantitative coronary angiography}

Sensitivity, specificity, PPV, NPV, and accuracy of CTA for the detection of coronary stenoses on QCA was $88 \%(95 \% \mathrm{Cl}$, 80-94\%), 95\% (92-98\%), 89\% (81-95\%), 95\% (91-97\%), and $93 \%$ (90-96\%), respectively, on vessel-based analysis, and 94\% (83-99\%), 100\% (88-100\%), 100\% (92-100\%), 91\% (75-98\%), and 96\% (89-99\%), respectively, on patient-based analysis.

\section{Assessment of flow-limiting stenoses}

On both combined analyses of CTA/MPI as well as QCA/MPI 19/78 (24\%) patients had flow-limiting stenoses. Eight stenoses were localized in the LAD-, 2 in the LCX-, and 9 in the RCA-territory. Sensitivity, specificity, PPV, NPV, and accuracy of the combination CTA/ MPI for the detection of flow-limiting coronary stenoses on QCA/ MPI was 100\% (95\% Cl, 82-100\%), 100\% (99-100\%), 100\% (82-100\%), 100\% (99-100\%), and 100\% (99-100\%), respectively, on vessel-based analysis, and 100\% (82-100\%), 100\% (94-100\%), $100 \%$ (82-100\%), 100\% (94-100\%), and 100\% (95-100\%), respectively, on patient-based analysis. When including fixed perfusion defects into the analysis, the PPV of MPI was $85 \%$ with a clear 


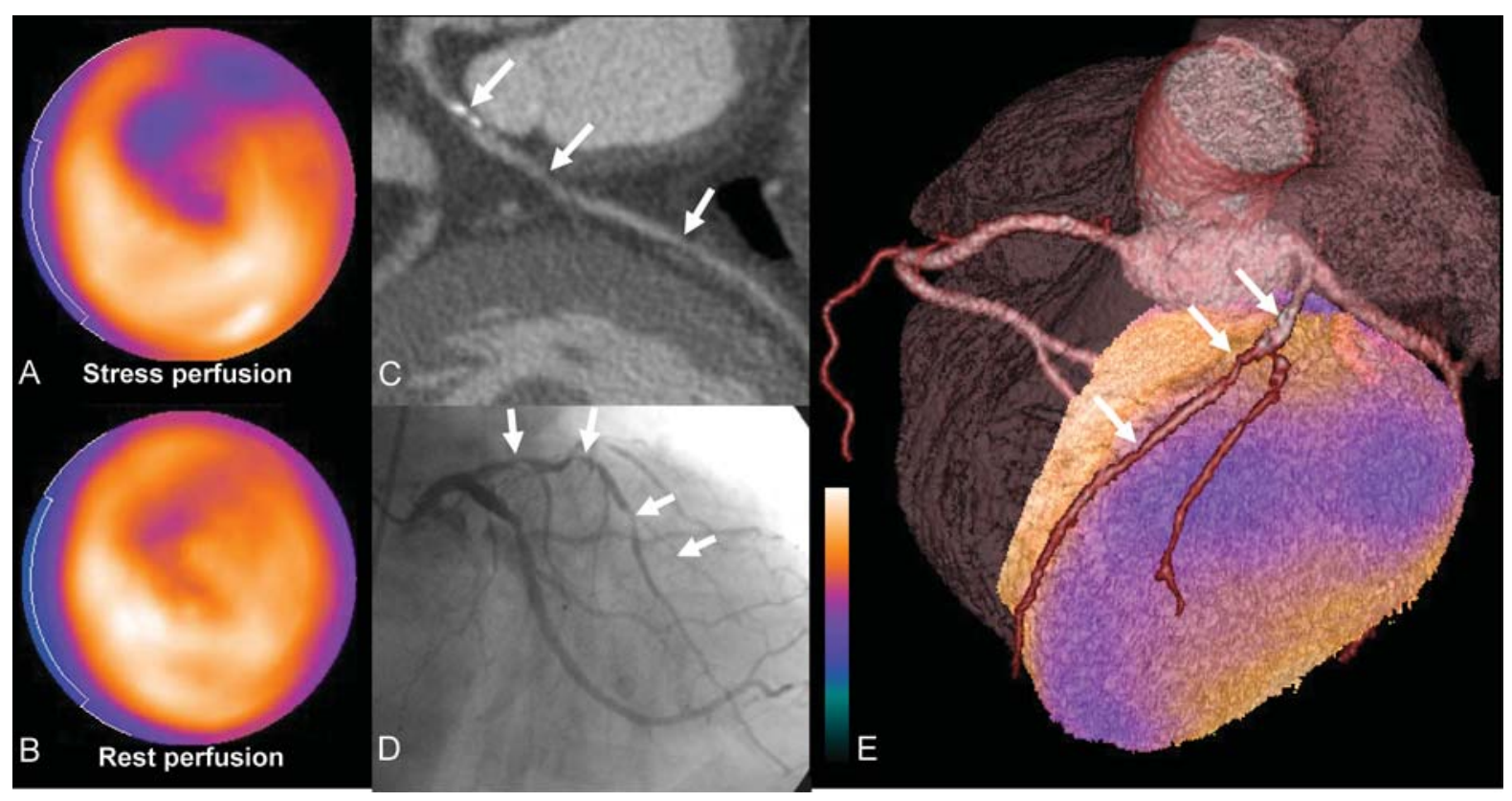

Figure 2 Myocardial perfusion SPECT after pharmacological stress $(A)$ and at rest $(B)$ showing a reversible anterior perfusion defect (ischaemia). The CT angiography multiplanar reconstruction of the left anterior descending artery (LAD) (C) shows three serial stenoses (arrows) confirmed by conventional coronary angiography ( $D$, arrows). ( $E)$ Three-dimensional SPECT/CT fusion images visualize matching of LAD stenoses (arrows) and anterior ischaemia.

trend towards improvement after addition of CTA (96\%) although the difference fell short of statistical significance $(P=0.07)$.

\section{Revascularizations}

A revascularization procedure was performed in 40/78 (51\%) patients of whom 11/78 (14\%) underwent CABG and 29/78 (37\%) $\mathrm{PCl}$ (with stenting in all but one patients). The median time interval between $\mathrm{CA}$ and revascularization procedure was 0 days (range, $0-51$ days), all $\mathrm{PCl}$ procedures were performed ad hoc. On vessel-based analysis, 62/310 (20\%) coronary arteries were revascularized (LMA, $n=2$; LAD, $n=23$; LCX, $n=21$; RCA, $n=16$ ).

Figure 3 shows the study population subcategorized according to the presence or absence of flow-limiting stenoses on CTA/MPI or QCA/MPI and the treatment strategy (revascularization vs. medical treatment) on patient- and vessel-based analysis. Typical angina was present in $21(53 \%)$ and $9(24 \%)$ patients $(P=0.02)$ in the revascularization and medical group, respectively, and a pathological exercise test in $16(40 \%)$ and $19(50 \%)$ patients, respectively $(P=0.51)$. However, an exercise test was only performed in 44 (56\%) of patients. Among the 21 patients without flow-limiting stenoses undergoing revascularization, the prevalence of symptoms was: typical angina, 25\% (10/21); atypical chest pain, 5\% (2/21); dyspnoea, 10\% (4/21); and no symptoms, $13 \%$ (5/21) ( $P=$ NS compared with patients without flow-limiting stenoses and medical treatment).

All patients with flow-limiting stenoses were revascularized. However, more than half of revascularization procedures (21/40, $53 \%)$ were performed in patients without flow-limiting stenoses and $76 \%(47 / 62)$ of revascularized vessels were not associated with ischaemia on MPI (Figure 4). Nineteen per cent (4/21) of revascularization procedures in patients without flow-limiting stenoses were CABG and $81 \%(17 / 21)$ ad hoc PCls. The fraction of CABG among patients without flow-limiting stenoses tended to be lower than in patients with flow-limiting stenoses (19 vs. $37 \%, P=N S)$. In patients with no flow-limiting stenoses, there were no differences in baseline characteristics between those undergoing revascularization $(n=21)$ and those treated medically $(n=38)$ except for a higher prevalence of known CAD in the former group (62 vs. $13 \%, P<0.001$ ).

Significant clinical predictors of revascularization by univariate logistic regression were a history of CAD, the presence of typical angina, history of diabetes mellitus, and current or former smoking status (Table 3). Multivariate logistic regression analysis identified a history of diabetes mellitus as the only independent predictor of revascularization.

\section{Discussion}

Our study documents an excellent ability of a combined noninvasive approach with CTA and MPI using SPECT for detecting flow-limiting coronary stenoses compared with the gold standard of QCA combined with MPI. In all patients with flow-limiting coronary stenoses (i.e. stenoses that were associated with myocardial ischaemia as evidenced by MPI), a revascularization procedure was performed. However, half of patients undergoing revascularization lacked any flow-limiting coronary stenoses based on non-invasive imaging and almost three quarters of revascularized vessels were 
Table 2 Imaging results

\begin{tabular}{|c|c|c|c|c|}
\hline & All patients $(n=78)$ & Revasc group $(n=40)$ & Medical group $(n=38)$ & $P$-value* \\
\hline \multicolumn{5}{|l|}{ CTA results } \\
\hline Coronary calcium score (ASE) ${ }^{\mathrm{a}}$ & $370(100,915)$ & $529(193,1432)$ & $156(1,586)$ & 0.001 \\
\hline Patients with CTA stenoses, $n(\%)$ & $46(59)$ & $39(98)$ & $7(18)$ & $<0.001$ \\
\hline LMA stenoses & $0(0)$ & $0(0)$ & $0(0)$ & - \\
\hline LAD stenoses & $33(42)$ & $30(75)$ & $3(8)$ & $<0.001$ \\
\hline LCX stenoses & $28(36)$ & $25(63)$ & $3(8)$ & $<0.001$ \\
\hline RCA stenoses & $30(38)$ & $26(65)$ & $4(11)$ & $<0.001$ \\
\hline \multicolumn{5}{|l|}{ MPI results } \\
\hline Fixed perfusion defects & $13(17)$ & $9(23)$ & $4(11)$ & 0.27 \\
\hline Reversible and partially reversible perfusion defects & $20(26)$ & $20(50)$ & $0(0)$ & $<0.001$ \\
\hline Normal MPI perfusion & $47(60)$ & $13(33)$ & $34(89)$ & $<0.001$ \\
\hline \multicolumn{5}{|l|}{ QCA results } \\
\hline Patients with QCA stenoses, $n(\%)$ & $49(63)$ & $40(100)$ & $9(24)$ & $<0.001$ \\
\hline LMA stenoses & $1(1)$ & $1(3)$ & $0(0)$ & 0.33 \\
\hline LAD stenoses & $33(42)$ & $27(68)$ & $6(16)$ & $<0.001$ \\
\hline LCX stenoses & $29(37)$ & $28(70)$ & $1(3)$ & $<0.001$ \\
\hline RCA stenoses & $29(37)$ & $26(65)$ & $3(8)$ & $<0.001$ \\
\hline Combination CTA/MPI: flow-limiting stenoses & $19(24)$ & $19(48)$ & $0(0)$ & $<0.001$ \\
\hline Combination QCA/MPI: flow-limiting stenoses & $19(24)$ & $19(48)$ & $0(0)$ & $<0.001$ \\
\hline
\end{tabular}

Revasc group, group of patients undergoing coronary revascularization; Medical group, group of patients treated conservatively; CTA, CT angiography; ASE, Agatston score equivalents; LMA, left main artery; LAD, left anterior descending artery; LCX, left circumflex artery; RCA, right coronary artery; MPI, myocardial perfusion imaging; QCA, quantitative coronary angiography.

${ }^{a}$ Data for coronary calcium score are given as median and interquartile range and comparison performed using Mann-Whitney $U$ test.

$*$ P-value for comparison of Revasc vs. Medical group.

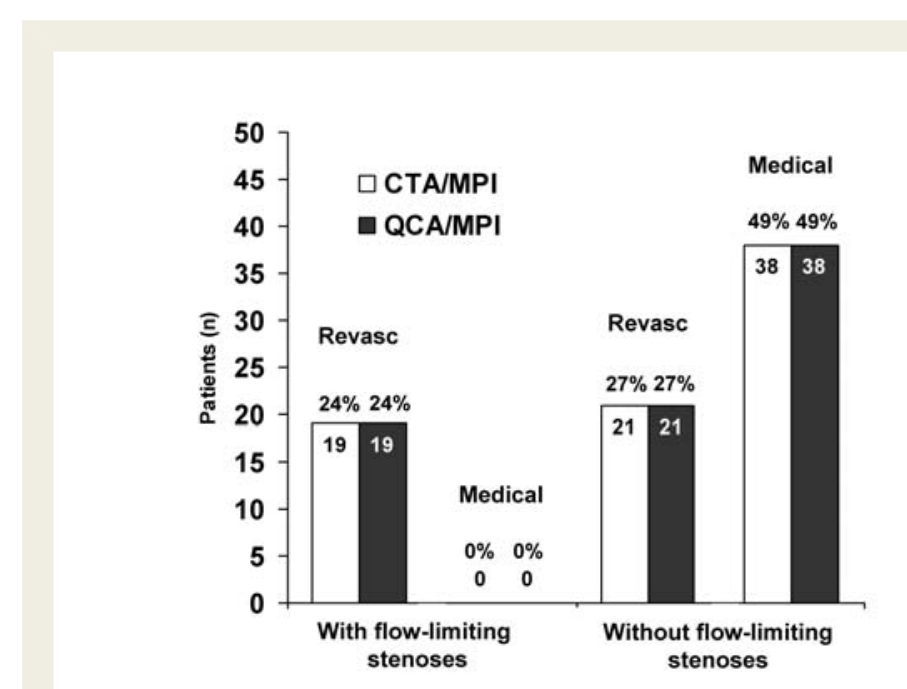

A. Patient-based analysis $(n=78)$

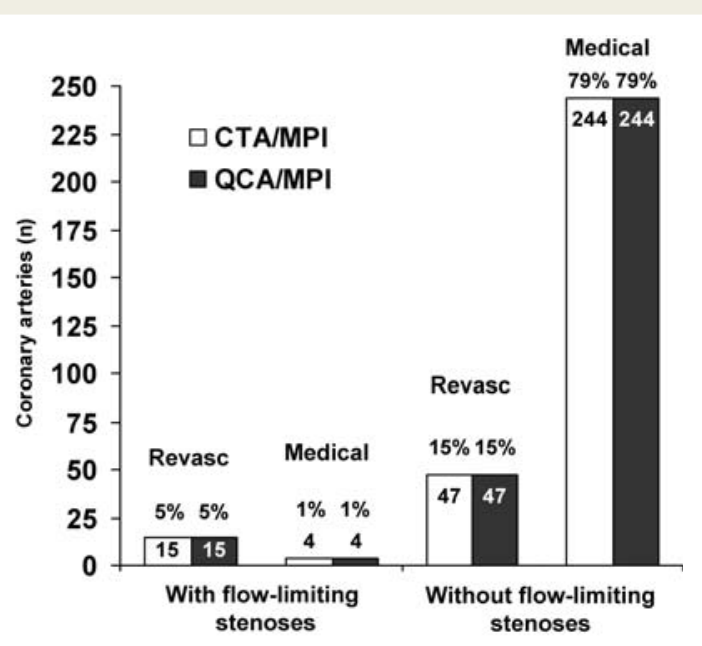

B. Vessel-based analysis $(n=310)$

Figure 3 Classification of patients (A. patient-based analysis) and coronary arteries (B. vessel-based analysis) according to the presence or absence of flow-limiting stenoses on the combination of CTA and MPI (CTA/MPI, empty bars) or QCA and MPI (QCA/MPI, filled bars) and the treatment strategy (Revasc, coronary revascularization; Medical, conservative treatment).

not associated with myocardial ischaemia. These findings underline a potential role of a combined non-invasive assessment with CTA and MPI as a gatekeeper for revascularization procedures in order to avoid its overuse and the associated burden of periprocedural morbidity. By doing so, in our study population in 21/78 (27\%) patients, an unnecessary revascularization procedure might have 


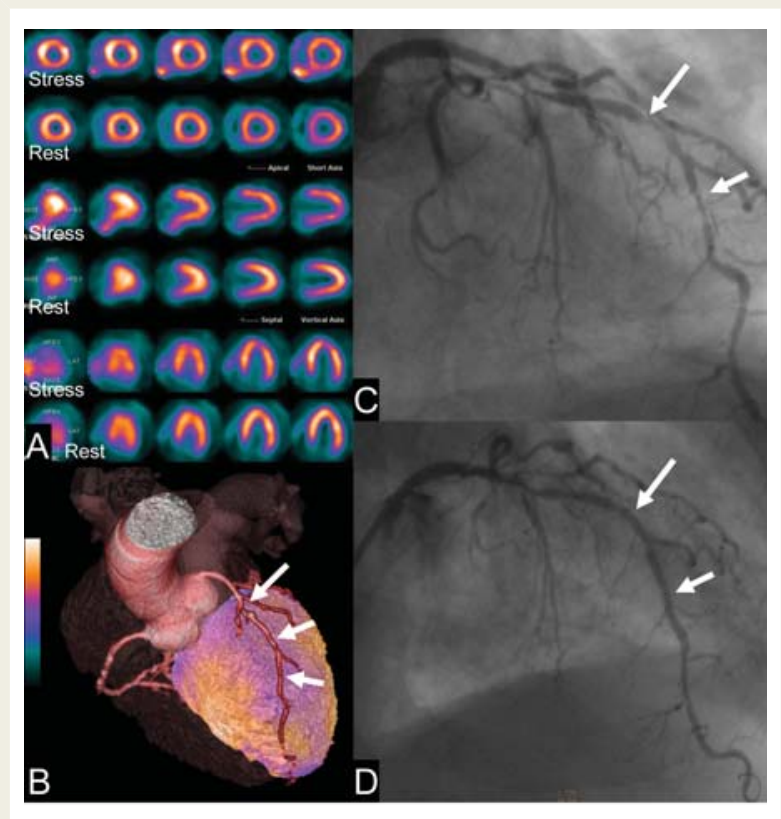

Figure 4 Example of a patient with non-flow-limiting stenoses undergoing revascularization. (A) Myocardial perfusion SPECT shows no significant perfusion defects during vasodilator stress or at rest. (B) The 3D SPECT/CT fusion images depict stenoses in the proximal, mid, and distal left anterior descending artery (LAD) (arrows). Invasive coronary angiography prior $(C)$ and after $\mathrm{PCl}(D)$ document stenting of the mid and distal LAD lesions (arrows). The proximal LAD lesion was left untreated.

Table 3 Univariate predictors of revascularization

\begin{tabular}{|c|c|c|}
\hline & OR $(95 \% \mathrm{Cl})$ & $P$-value* \\
\hline \multicolumn{3}{|l|}{ Clinical characteristics } \\
\hline Age (years) & $1.04(0.98-1.09)$ & 0.18 \\
\hline History of CAD & $3.55(1.13-11.15)$ & 0.03 \\
\hline Previous MI/ACS & $2.50(0.78-8.06)$ & 0.12 \\
\hline Pathological exercise test & $0.67(0.27-1.64)$ & 0.38 \\
\hline $\operatorname{LVEF}(\%)$ & $1.00(0.97-1.03)$ & 0.94 \\
\hline \multicolumn{3}{|l|}{ Symptoms } \\
\hline Angina pectoris CCS I-III & $3.56(1.35-9.41)$ & 0.01 \\
\hline Atypical chest pain & $0.35(0.11-1.13)$ & 0.08 \\
\hline Dyspnoea NYHA I-III & $0.46(0.16-1.33)$ & 0.15 \\
\hline None & $1.13(0.34-3.73)$ & 0.84 \\
\hline \multicolumn{3}{|l|}{ Cardiovascular risk factors } \\
\hline Diabetes mellitus & $6.83(1.40-33.28)$ & 0.02 \\
\hline Hypertension & $2.31(0.76-7.05)$ & 0.14 \\
\hline Dyslipidaemia & $2.06(0.84-5.09)$ & 0.12 \\
\hline Current or former smoker & $2.56(1.03-6.37)$ & 0.04 \\
\hline $\begin{array}{l}\mathrm{OR} \text {, odds ratio; } \mathrm{Cl} \text {, confidence inter } \\
\text { myocardial infarction; } \mathrm{ACS} \text {, acute cc } \\
\text { ejection fraction. }\end{array}$ & $\begin{array}{l}\text { AD, coronary artery } \\
\text { ry syndrome; LVEF, I }\end{array}$ & $\begin{array}{l}\text { ase; Ml, } \\
\text { ntricular }\end{array}$ \\
\hline
\end{tabular}

been prevented, while in none of the patients a revascularization procedure would have been falsely withheld.

The role of revascularization procedures in patients with stable CAD is controversial. As with medical therapy, the objectives of coronary revascularization procedures are two-fold, to improve survival free of ischaemic events, and to diminish or eradicate ischaemic symptoms. ${ }^{4}$ Since the highest risk patients derive the highest benefit from revascularization procedures, both the individual risk of the patient as well as his symptomatic status must be a major determinant in the decision-making process. High risk factors include high risk angiographical configuration (LMA disease, proximal three-vessel disease), impaired left ventricular function, pronounced symptoms (angina CCS III-IV), or the presence of myocardial ischaemia by non-invasive testing. ${ }^{19}$ Coronary revascularization procedures have convincingly shown to reduce ischaemic symptoms and improve quality of life even in patients at low risk. ${ }^{7}$ However, periprocedural morbidity and mortality remain important considerations. In fact, in a stable CAD population, coronary revascularization procedures have failed to demonstrate an improvement in prognosis compared with state-of-the-art medical therapy. ${ }^{5,7,19,20}$ Therefore, guidelines recommend proof of ischaemia prior to revascularization procedures. ${ }^{2,4}$ If moderate to large ischaemia is present, however, a coronary revascularization may actually improve prognosis compared with medical treatment ${ }^{21}$ by a more effective reduction in the amount of jeopardized myocardium. $^{22}$ On the other hand, revascularization of a non-flow-limiting coronary stenosis is not of benefit for the patient, neither from a prognostic nor from a symptomatic point of view. ${ }^{23,24}$

Therefore, MPI has been suggested as gatekeeper for invasive examination. ${ }^{25}$ However, combining MPI with CTA has been shown to improve the accuracy of non-invasive assessment, ${ }^{26}$ as false positive MPI findings may be disproved by negative CTA which may be of particular importance in low CAD prevalence populations. In addition, not every flow-limiting lesion may be suitable for $\mathrm{PCl}$, which may be identified by CTA avoiding futile invasive attempts. Interestingly, only $56 \%$ of patients in our nonselected study population had undergone a stress ECG prior to CA, and in less than half of patients the stress ECG was pathologic. However, patients in the revascularization group had a higher prevalence of angina pectoris. This may explain why a considerable amount of presumably non-flow-limiting lesions were nevertheless revascularized, as $87 \%$ of these patients had symptoms. This reflects that in clinical reality, a decision to revascularize often also incorporates the overall presentation and symptoms of a patient. Of note, the vast majority of these revascularization procedures were ad hoc PCls. This observation is in line with previous publications reporting that $66 \%$ of $\mathrm{PCls}$ are ad hoc procedures in stable CAD patients. ${ }^{27}$

Several factors may contribute to the observed discrepancy between guidelines and the use of $\mathrm{PCl}$ in real life, among them insufficient use of non-invasive testing, and potential medicolegal considerations (presumably not allowing a detected stenosis untreated). The latter may be driven by the open artery theory, although not supported by the latest results. ${ }^{28}$ In fact, previous reports have documented a high correlation between catheterization and revascularization rates. ${ }^{29}$ Our results suggest the use of a combined non-invasive approach with CTA and MPI prior to 
coronary revascularization as a method to help providers more fully incorporate clinical evidence into their decision-making process. Additionally, a comprehensive non-invasive assessment of CAD with CTA and MPI would allow for careful selection of the optimal revascularization procedure according to the guidelines improving the balance between periprocedural risk and prognostic benefit for each individual patient.

\section{Study limitations}

An important drawback of non-invasive cardiac imaging is the high radiation exposure associated with CTA and MPI. In fact, studies with 64-slice CTA reported an estimated radiation burden of up to $21.4 \mathrm{mSv}$ without the use of ECG-pulsing ${ }^{30}$ and MPI-SPECT studies with ${ }^{99 \mathrm{~m}} \mathrm{Tc}$-based radiotracers are associated with radiation doses in the range of $9-11 \mathrm{mSv} .^{31}$ However, with the implementation of prospective ECG-gating protocols for CTA, radiation exposure can be reduced down to $2.1 \mathrm{mSv}^{32,33}$ and alternative MPI techniques such as positron emission tomography with ${ }^{13} \mathrm{~N}$-ammonia or ${ }^{15} \mathrm{O}$-water may reduce radiation placing the resulting radiation exposure well within the range of conventional CA. Additionally, hybrid imaging combining CTA using prospective ECG gating with stress-only SPECT has been suggested as an attractive alternative to standard stress/rest SPECT for the detection of $C A D$ reducing radiation exposure to $5.4 \mathrm{mSv}^{34}$

In the present study, we have defined flow-limiting stenoses as angiographically determined coronary narrowings associated with a reversible perfusion defect in the subtended myocardial territory. The perfusion defect was assessed by SPECT MPI, a method which may be limited to accurately localize ischaemia-producing lesions in patients with multivessel CAD. ${ }^{35}$ Thus, in these patients, determining which lesions warrant stenting can be difficult. This may potentially be overcome by invasively assessed fractional flow reserve. ${ }^{24}$ Whether alternative measures such as, for instance, cardiac magnetic resonance imaging with an in-plane resolution superior to SPECT may help solving this issue, remains to be determined.

Another shortcoming of our study was the limited number of study participants. As a result, no significant increments in diagnostic accuracy with CTA/MPI compared with MPI alone were observed. Furthermore, it was not possible to perform subgroup analysis across different patient strata such as patients with multivessel vs. patients with single vessel disease. In addition, the study was not designed to assess the clinical follow up. Therefore, a potential improvement in clinical symptomatology justifying the revascularization was possibly missed.

\section{Conclusions}

The combined non-invasive approach with CTA and MPI in patients with known or suspected CAD has an excellent accuracy to detect flow-limiting coronary stenoses compared with the gold standard of QCA combined with MPI and may be used as a gatekeeper for $\mathrm{CA}$ and revascularization procedures.

\section{Funding}

This study was supported by a European Society of Cardiology (ESC) research grant (O.G.), and by a grant from the Swiss National Science Foundation (P.A.K.).

\section{Conflict of interest: none declared.}

\section{References}

1. Topol EJ, Nissen SE. Our preoccupation with coronary luminology. The dissociation between clinical and angiographic findings in ischemic heart disease. Circulation 1995;92:2333-2342.

2. Smith SC Jr, Feldman TE, Hirshfeld JW Jr, Jacobs AK, Kern MJ, King SB 3rd, Morrison DA, O'Neill WW, Schaff HV, Whitlow PL, Williams DO, Antman EM, Adams CD, Anderson JL, Faxon DP, Fuster V, Halperin JL, Hiratzka LF, Hunt SA, Nishimura R, Ornato JP, Page RL, Riegel B. ACC/AHA/ SCAI 2005 guideline update for percutaneous coronary intervention: a report of the American College of Cardiology/American Heart Association Task Force on Practice Guidelines (ACC/AHA/SCAI Writing Committee to Update the 2001 Guidelines for Percutaneous Coronary Intervention). J Am Coll Cardiol 2006;47:e1-e121.

3. White CW, Wright CB, Doty DB, Hiratza LF, Eastham CL, Harrison DG, Marcus ML. Does visual interpretation of the coronary arteriogram predict the physiologic importance of a coronary stenosis? N Engl J Med 1984;310:819-824.

4. Fox K, Garcia MA, Ardissino D, Buszman P, Camici PG, Crea F, Daly C, De Backer G, Hjemdahl P, Lopez-Sendon J, Marco J, Morais J, Pepper J, Sechtem U, Simoons M, Thygesen K, Priori SG, Blanc JJ, Budaj A, Camm J, Dean V, Deckers J, Dickstein K, Lekakis J, McGregor K, Metra M, Osterspey A, Tamargo J, Zamorano JL. Guidelines on the management of stable angina pectoris: executive summary: the Task Force on the Management of Stable Angina Pectoris of the European Society of Cardiology. Eur Heart J 2006;27:1341-1381.

5. Boden WE, O'Rourke RA, Teo KK, Hartigan PM, Maron DJ, Kostuk WJ, Knudtson M, Dada M, Casperson P, Harris CL, Chaitman BR, Shaw L, Gosselin G, Nawaz S, Title LM, Gau G, Blaustein AS, Booth DC, Bates ER, Spertus JA, Berman DS, Mancini GB, Weintraub WS. Optimal medical therapy with or without $\mathrm{PCl}$ for stable coronary disease. N Engl J Med 2007;356: $1503-1516$

6. Gibbons RJ, Abrams J, Chatterjee K, Daley J, Deedwania PC, Douglas JS, Ferguson TB Jr, Fihn SD, Fraker TD Jr, Gardin JM, O'Rourke RA, Pasternak RC, Williams SV. ACC/AHA 2002 guideline update for the management of patients with chronic stable angina-summary article: a report of the American College of Cardiology/American Heart Association Task Force on practice guidelines (Committee on the Management of Patients With Chronic Stable Angina). J Am Coll Cardiol 2003;41:159-168.

7. Bucher HC, Hengstler P, Schindler C, Guyatt GH. Percutaneous transluminal coronary angioplasty versus medical treatment for non-acute coronary heart disease: meta-analysis of randomised controlled trials. Br Med J 2000;321:73-77.

8. Windecker S, Remondino A, Eberli FR, Juni P, Raber L, Wenaweser P, Togni M, Billinger M, Tuller D, Seiler C, Roffi M, Corti R, Sutsch G, Maier W, Lüscher T, Hess OM, Egger M, Meier B. Sirolimus-eluting and paclitaxel-eluting stents for coronary revascularization. N Engl J Med 2005;353:653-662.

9. Leschka S, Alkadhi H, Plass A, Desbiolles L, Grunenfelder J, Marincek B, Wildermuth S. Accuracy of MSCT coronary angiography with 64-slice technology: first experience. Eur Heart J 2005;26:1482-1487.

10. Scheffel H, Alkadhi H, Plass A, Vachenauer R, Desbiolles L, Gaemperli O, Schepis T, Frauenfelder T, Schertler T, Husmann L, Grunenfelder J, Genoni M, Kaufmann PA, Marincek B, Leschka S. Accuracy of dual-source CT coronary angiography: first experience in a high pre-test probability population without heart rate control. Eur Radiol 2006;16:2739-2747.

11. Gaemperli O, Schepis T, Valenta I, Husmann L, Scheffel H, Duerst V, Eberli FR, Lüscher TF, Alkadhi H, Kaufmann PA. Cardiac image fusion from stand-alone SPECT and CT: clinical experience. J Nucl Med 2007;48:696-703.

12. Gaemperli O, Schepis T, Valenta I, Koepfli P, Husmann L, Scheffel H, Leschka S, Eberli FR, Lüscher TF, Alkadhi H, Kaufmann PA. Functionally relevant coronary artery disease: comparison of 64-section CT angiography with myocardial perfusion SPECT. Radiology 2008;248:414-423.

13. Husmann L, Leschka S, Desbiolles L, Schepis T, Gaemperli O, Seifert B, Cattin P, Frauenfelder T, Flohr TG, Marincek B, Kaufmann PA, Alkadhi H. Coronary artery motion and cardiac phases: dependency on heart rate-implications for CT image reconstruction. Radiology 2007;245:567-576.

14. Austen WG, Edwards JE, Frye RL, Gensini GG, Gott VL, Griffith LS, McGoon DC, Murphy ML, Roe BB. A reporting system on patients evaluated for coronary artery disease. Report of the Ad Hoc Committee for Grading of Coronary Artery Disease, Council on Cardiovascular Surgery, American Heart Association. Circulation 1975;51:5-40.

15. Hesse B, Tagil K, Cuocolo A, Anagnostopoulos C, Bardies M, Bax J, Bengel F, Busemann Sokole E, Davies G, Dondi M, Edenbrandt L, Franken P, Kjaer A, Knuuti J, Lassmann M, Ljungberg M, Marcassa C, Marie PY, McKiddie F, O'Connor M, Prvulovich E, Underwood R, van Eck-Smit B. EANM/ESC procedural guidelines for myocardial perfusion imaging in nuclear cardiology. Eur J Nucl Med Mol Imaging 2005;32:855-897. 
16. Germano G, Kavanagh PB, Waechter P, Areeda J, Van Kriekinge S, Sharir T, Lewin HC, Berman DS. A new algorithm for the quantitation of myocardial perfusion SPECT. I: technical principles and reproducibility. I Nucl Med 2000;41: $712-719$.

17. Fleischmann S, Koepfli P, Namdar M, Wyss CA, Jenni R, Kaufmann PA. Gated (99m)Tc-tetrofosmin SPECT for discriminating infarct from artifact in fixed myocardial perfusion defects. J Nud Med 2004;45:754-759.

18. Diamond GA, Forrester JS. Analysis of probability as an aid in the clinical diagnosis of coronary-artery disease. N Engl J Med 1979;300:1350-1358.

19. Yusuf S, Zucker D, Peduzzi P, Fisher LD, Takaro T, Kennedy JW, Davis K, Killip T, Passamani E, Norris R for the Coronary Artery Bypass Graft Surgery Trialists. Effect of coronary artery bypass graft surgery on survival: overview of 10-year results from randomised trials by the Coronary Artery Bypass Graft Surgery Trialists Collaboration. Lancet 1994;344:563-570.

20. Pitt B, Waters D, Brown WV, van Boven AJ, Schwartz L, Title LM, Eisenberg D, Shurzinske L, McCormick LS. Aggressive lipid-lowering therapy compared with angioplasty in stable coronary artery disease. Atorvastatin versus Revascularization Treatment Investigators. N Engl J Med 1999;341:70-76.

21. Hachamovitch R, Hayes SW, Friedman JD, Cohen I, Berman DS. Comparison of the short-term survival benefit associated with revascularization compared with medical therapy in patients with no prior coronary artery disease undergoing stress myocardial perfusion single photon emission computed tomography. Circulation 2003;107:2900-2907.

22. Shaw LJ, Berman DS, Maron DJ, Mancini GB, Hayes SW, Hartigan PM, Weintraub WS, O'Rourke RA, Dada M, Spertus JA, Chaitman BR, Friedman J, Slomka P, Heller GV, Germano G, Gosselin G, Berger P, Kostuk WJ, Schwartz RG, Knudtson M, Veledar E, Bates ER, McCallister B, Teo KK, Boden WE. Optimal medical therapy with or without percutaneous coronary intervention to reduce ischemic burden: results from the Clinical Outcomes Utilizing Revascularization and Aggressive Drug Evaluation (COURAGE) trial nuclear substudy. Circulation 2008;117:1283-1291.

23. Pijls NH, van Schaardenburgh P, Manoharan G, Boersma E, Bech JW, van't Veer M, Bar F, Hoorntje J, Koolen J, Wijns W, de Bruyne B. Percutaneous coronary intervention of functionally nonsignificant stenosis: 5-year follow-up of the DEFER Study. J Am Coll Cardiol 2007;49:2105-2111.

24. Tonino PA, De Bruyne B, Pijls NH, Siebert U, Ikeno F, van't Veer M, Klauss V, Manoharan G, Engstrom T, Oldroyd KG, Ver Lee PN, MacCarthy PA, Fearon WF. Fractional flow reserve versus angiography for guiding percutaneous coronary intervention. N Engl J Med 2009;360:213-224.

25. Hoilund-Carlsen PF, Johansen A, Christensen HW, Vach W, Moldrup M, Bartram P, Veje A, Haghfelt T. Potential impact of myocardial perfusion scintigraphy as gatekeeper for invasive examination and treatment in patients with stable angina pectoris: observational study without post-test referral bias. Eur Heart J 2006;27:29-34.

26. Rispler S, Keidar Z, Ghersin E, Roguin A, Soil A, Dragu R, Litmanovich D, Frenkel A, Aronson D, Engel A, Beyar R, Israel O. Integrated single-photon emission computed tomography and computed tomography coronary angiography for the assessment of hemodynamically significant coronary artery lesions. J Am Coll Cardiol 2007;49:1059-1067.

27. Cook S, Walker A, Hugli O, Togni M, Meier B. Percutaneous coronary interventions in Europe: prevalence, numerical estimates, and projections based on data up to 2004. Clin Res Cardiol 2007; 96:375-382.

28. Hochman JS, Lamas GA, Buller CE, Dzavik V, Reynolds HR, Abramsky SJ, Forman S, Ruzyllo W, Maggioni AP, White H, Sadowski Z, Carvalho AC, Rankin JM, Renkin JP, Steg PG, Mascette AM, Sopko G, Pfisterer ME, Leor J, Fridrich V, Mark DB, Knatterud GL. Coronary intervention for persistent occlusion after myocardial infarction. N Engl J Med 2006;355:2395-2407.

29. Verrilli D, Welch HG. The impact of diagnostic testing on therapeutic interventions. JAMA 1996;275:1189-1191.

30. Mollet NR, Cademartiri F, van Mieghem CA, Runza G, McFadden EP, Baks T, Serruys PW, Krestin GP, de Feyter PJ. High-resolution spiral computed tomography coronary angiography in patients referred for diagnostic conventional coronary angiography. Circulation 2005;112:2318-2323.

31. Einstein AJ, Moser KW, Thompson RC, Cerqueira MD, Henzlova MJ. Radiation dose to patients from cardiac diagnostic imaging. Circulation 2007;116:1290-1305.

32. Husmann L, Valenta I, Gaemperli O, Adda O, Treyer V, Wyss CA, Veit-Haibach P, Tatsugami F, von Schulthess GK, Kaufmann PA. Feasibility of low-dose coronary CT angiography: first experience with prospective ECG-gating. Eur Heart J 2008;29:191-197.

33. Herzog BA, Husmann L, Valenta I, Tay FM, Burkhard N, Gaemperli O, Wyss CA, Landmesser $U$, Kaufmann PA. Determinants of vessel contrast in BMI-adapted low dose CT coronary angiography with prospective ECG-triggering. Int J Cardiovasc Imaging 2009;25:625-630.

34. Husmann L, Herzog BA, Gaemperli O, Tatsugami F, Burkhard N, Valenta I, Veit-Haibach P, Wyss CA, Landmesser U, Kaufmann PA. Diagnostic accuracy of computed tomography coronary angiography and evaluation of stress-only singlephoton emission computed tomography/computed tomography hybrid imaging: comparison of prospective electrocardiogram-triggering vs. retrospective gating. Eur Heart J 2009;30:600-607.

35. Lima RS, Watson DD, Goode AR, Siadaty MS, Ragosta M, Beller GA, Samady H. Incremental value of combined perfusion and function over perfusion alone by gated SPECT myocardial perfusion imaging for detection of severe three-vessel coronary artery disease. J Am Coll Cardiol 2003;42:64-70. 